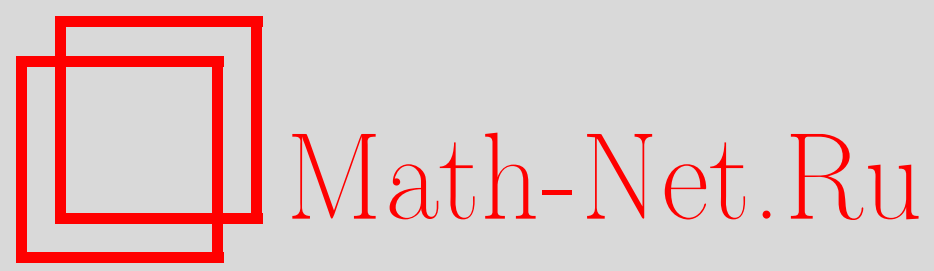

Ю. Е. Егорова, K методу решения задач возможностно-вероятностного программирования, Нечеткие системы и мягкие вычисления, 2021, том 16, выпуск $1,21-33$

DOI: https://doi.org/10.26456/fssc77

Использование Общероссийского математического портала Math-Net.Ru подразумевает, что вы прочитали и согласны с пользовательским соглашением

http: //www. mathnet.ru/rus/agreement

Параметры загрузки:

IP : 18.208 .226 .222

26 апреля 2023 г., 16:04:00 
УДК 510.676, 519.7

\section{К МЕТОДУ РЕШЕНИЯ ЗАДАЧ ВОЗМОЖНОСТНО-ВЕРОЯТНОСТНОГО ПРОГРАММИРОВАНИЯ ${ }^{1}$}

Егорова Ю.Е.

Тверской государственный университет, г. Тверь

Поступила в редакцию 25.12.2020, после переработки 06.05.2021.

В статье исследуется задача возможностно-вероятностной оптимизации, основанная на принципе ожидаемой возможности, и метод решения ее стохастического аналога в случае слабейшей $\mathrm{t}$-нормы, описывающей взаимодействие нечетких параметров. Получены более простые для проверки условия, обеспечивающие сходимость метода стохастических квазиградиентов решения эквивалентного стохастического аналога.

Ключевые слова: возможностно-вероятностная оптимизация, метод стохастических квазиградиентов, нечеткая случайная величина, слабейшая $\mathrm{t}$-норма.

Нечеткие системы и млгкие вычисления. 2021. Т. 16, № 1. С. 21-33. https://doi.org/10.26456/fssc77

\section{1. Введение}

В настоящее время задачи возможностно-вероятностной оптимизации, в которых взаимодействие нечетких параметров описывается слабейшей t-нормой, представляют большой интерес как с теоретической [1-7], так и с практической точек зрения $[8,9]$. Одним из применений таких задач является оптимизация инвестиционного портфеля в условиях гибридной неопределенности $[8,9]$.

Для решения задач возможностно-вероятностной оптимизации были разработаны различные методы. Одни $[1,2,8]$ основаны на непрямом подходе, сводящем задачу к эквивалентной детерминированной, которая в последствие решается известными методами оптимизации. Другим подходом является сведение задачи к стохастическому эквиваленту и применение методов стохастических квазиградиентов $[3,4,6]$ или стохастического метода штрафов $[7,9]$.

Однако, в случае методов, использующих стохастические квазиградиенты, представленные теоремы о сходимости этих методов $[3,4,7]$ включают условия, которые не всегда удобно проверять на практике. Поэтому целью данной статьи является показать, что теоремы о сходимости остаются справедливыми, если некоторые их условия ослабить, заменив на более простые для проверки в реальных задачах.

\footnotetext{
${ }^{1}$ Работа выполнена при финансовой поддержке РФФИ (проект №20-01-00669).

(C) Егорова Ю.Е., 2021
} 


\section{2. Необходимые понятия и обозначения}

В контексте работ [10-17] введем ряд определений и понятий из теории возможностей. Пусть $(\Gamma, \mathfrak{P}(\Gamma), \pi)$ и $(\Omega, \mathcal{B}, P)$ есть возможностное и вероятностное пространства, в которых $\Omega-$ пространство элементарных событий $\omega \in \Omega, \Gamma-$ модельное пространство с элементами $\gamma \in \Gamma, \mathcal{B}-\sigma$-алгебра событий, $\mathfrak{P}(\Gamma)-$ множество всех подмножеств $\Gamma, \pi-$ мера возможности, а $P$ - мера вероятности; $E^{1}$ - числовая прямая.

Дадим определение нечеткой случайной (возможностно-вероятностной) величины и ее распределения $[11,12]$.

Определение 1. Нечеткая случайная величина $Y$ есть вещественная функиия $Y: \Omega \times \Gamma \rightarrow \mathrm{E}^{1}$, -измеримая для каждого фиксированного $\gamma$, а функиия

$$
\mu_{Y}(\omega, t)=\pi\{\gamma \in \Gamma: Y(\omega, \gamma)=t\}
$$

называется ее функиией распределения.

Из определения 1 следует, что функция распределения нечеткой случайной величины зависит от случайного параметра, т. е. является случайной функцией.

Определение 2. Пусть $Y(\omega, \gamma)$ - нечеткая случайная величина. Её ожидаемым значением $E[Y][11,12]$ называется нечеткая величина, имеющая функиию распределения возможностей

$$
\mu_{\mathrm{E}[Y]}(t)=\pi\{\gamma \in \Gamma: \mathrm{E}[Y(\omega, \gamma)]=t\}
$$

где $\mathrm{E}[\cdot]$ есть оператор взятия математического ожидания

$$
\mathrm{E}[Y(\omega, \gamma)]=\int_{\Omega} Y(\omega, \gamma) \operatorname{Pd} \omega .
$$

В данном случае функция распределения ожидаемого значения нечеткой случайной величины уже не зависит от случайного параметра и является детерминированной.

Определяемое в соответствии с рассматриваемым подходом математическое ожидание случайных величин наследует основные свойства вещественных случайных величин.

Интересным для приложений является сдвиг-масштабное представление [14] нечетких случайных величин:

$$
Y(\omega, \gamma)=a(\omega)+\sigma(\omega) Z(\gamma)
$$

где $a(\omega)$ и $\sigma(\omega)$ - случайные величины, определенные на $(\Omega, \mathcal{B}, P)$, а $Z(\gamma)$ есть нечеткая величина, определенная на $(\Gamma, P(\Gamma), \pi)$. В данном случае, $a(\omega)$ и $\sigma(\omega)$ обозначают соответственно параметры сдвига и масштаба.

Для моделирования нечетких величин часто используется распределение LRтипа [15].

Определение 3. Нечеткая величина $Z$ называется величиной LR-типа, если ее функиия распределения возможности имеет вид

$$
\mu_{Z}(t)= \begin{cases}L\left(\frac{\underline{\underline{m}}-t}{\underline{d}}\right), & t<\underline{m}, \\ 1, & \underline{m} \leq t \leq \bar{m}, \\ R\left(\frac{t-\bar{m}}{\bar{d}}\right), & t>\bar{m},\end{cases}
$$


где $L(t)$ и $R(t)$ - функиии представления формы. В этом случае величина $Z$ записывается, как правило, в виде $Z=[\underline{m}, \bar{m}, \underline{d}, \bar{d}]_{L R}$.

В данном представлении, $\underline{m}$ и $\bar{m}$ есть левая и правая границы интервала толерантности, a $\underline{d}, \bar{d}-$ коэффициенты нечеткости; при этом $\underline{m} \leq \bar{m}$ и $\underline{d}>0, \bar{d}>0$.

Для агрегирования нечеткой информации будем использовать t-нормы и tконормы, которые расширяют операции типа min и $\max$, заложенные в действиях над нечеткими множествами и нечеткими величинами [16].

Особый интерес представляют t-нормы

$$
T_{M}(x, y)=\min \{x, y\} \text { и } T_{W}(x, y)= \begin{cases}\min \{x, y\}, & \text { если } \max \{x, y\}=1 \\ 0, & \text { иначе }\end{cases}
$$

которые являются экстремальными.

Сильнейшей t-нормой называется t-норма $T_{M}$, a $T_{W}-$ слабейшей t-нормой, так как для любой произвольной t-нормы $T$ и $\forall x, y \in[0,1]$ справедливо неравенство

$$
T_{W}(x, y) \leq T(x, y) \leq T_{M}(x, y) .
$$

Опираясь на результаты работы [17], обобщим понятие несвязанности нечетких величин, введенное в [10], на случай произвольной t-нормы.

Определение 4. Множества $A_{1}, \ldots, A_{n} \in P(\Gamma)$ называются взаимно Т связанными, если для любого индексного множества $\left\{i_{1}, \ldots i_{k}\right\} \subset\{1, \ldots, n\}, 1 \leq k \leq n$ справедливо

$$
\pi\left(X_{i_{1}} \cap \cdots \cap X_{i_{k}}\right)=T\left(\pi\left(X_{i_{1}}\right), \ldots, \pi\left(X_{i_{k}}\right)\right)
$$

əдe

$$
\begin{aligned}
T\left(\pi\left(A_{i_{1}}\right), \ldots, \pi\left(A_{i_{k}}\right)\right)= & \\
& T\left(T\left(\ldots T\left(T\left(\pi\left(A_{i_{1}}, \pi\left(A_{i_{2}}\right)\right), \pi\left(A_{i_{3}}\right)\right), \ldots \pi\left(A_{i_{k-1}}\right)\right), \pi\left(A_{i_{k}}\right)\right) .\right.
\end{aligned}
$$

Пусть $Z_{1}, \ldots, Z_{n}$ есть нечеткие величины, определенные на возможностном пространстве $(\Gamma, P(\Gamma), \pi)$. Перенесем понятие взаимной Т-связанности множеств (событий) на нечеткие величины.

Определение 5. Нечеткие величины $Z_{1}, \ldots, Z_{n}$ называются взаимно $T$ связанными, если для любого индексного множества $\left\{i_{1}, \ldots i_{k}\right\} \subset\{1, \ldots, n\}, 1 \leq k \leq n$ справедливо

$$
\begin{aligned}
\mu_{Z_{i_{1}}, \ldots, Z_{i_{k}}}\left(x_{i_{1}}, \ldots, x_{i_{k}}\right) & =\pi\left\{\gamma \in \Gamma: Z_{i_{1}}(\gamma)=x_{i_{1}}, \ldots, Z_{i_{k}}(\gamma)=x_{i_{k}}\right\} \\
& =\pi\left\{Z_{i_{1}}^{-1}\left\{x_{i_{1}}\right\} \cap \cdots \cap Z_{i_{k}}^{-1}\left\{x_{i_{k}}\right\}\right\} \\
& =T\left(\pi\left(Z_{i_{1}}^{-1}\left\{x_{i_{1}}\right\}\right), \ldots, \pi\left(Z_{i_{k}}^{-1}\left\{x_{i_{k}}\right\}\right)\right), \quad x_{i_{j}} \in E^{1} .
\end{aligned}
$$

\section{3. Модель возможностно-вероятностной оптимизации}

В статье будем рассматривать следующую модель возможностновероятностной оптимизации, сформулированную впервые для случая дискретных вероятностных распределений в [1]: 


$$
\begin{gathered}
k \rightarrow \underset{x}{\text { extr, }}, \\
\tau_{0}\left\{\mathrm{E}\left[f_{0}(x, \omega, \gamma)\right] \mathcal{R}_{0} k\right\} \geq \alpha_{0}, \\
\left\{\begin{array}{l}
\tau_{i}\left\{\mathrm{E}\left[f_{i}(x, \omega, \gamma)\right] \mathcal{R}_{i} 0\right\} \geq \alpha_{i}, i=\overline{1, m} \\
x \in X .
\end{array}\right.
\end{gathered}
$$

В представленной модели $X \subseteq \mathrm{E}_{+}^{n}=\left\{x \in E^{n}: x \geq 0\right\} ; f_{i}(x, \omega, \gamma)$ есть нечеткие случайные функции

$$
f_{i}: X \times \Omega \times \Gamma \rightarrow E^{1}, i=\overline{0, m} ;
$$

$\tau_{i}$ есть мера возможности $\pi$ или мера необходимости $\nu ; \mathcal{R}_{0}, \mathcal{R}_{i}-$ четкие отношения $\{\leq, \geq,=\} ; \alpha_{i} \in(0,1], k$ - дополнительные переменные.

Пусть функции $f_{i}(x, \omega, \gamma), i=\overline{0, m}$ имеют вид

$$
f_{i}(x, \omega, \gamma)=\sum_{j=0}^{n} b_{i j}(\omega, \gamma) x_{j}-b_{i}(\omega, \gamma)
$$

где нечеткие случайные величины $b_{i j}(\omega, \gamma), b_{i}(\omega, \gamma)$ имеют сдвиг-масштабное представление

$$
\begin{gathered}
b_{i j}(\omega, \gamma)=a_{i j}(\omega)+\sigma_{i j}(\omega) Z_{i j}(\gamma), \\
b_{i}(\omega, \gamma)=a_{i}(\omega)+\sigma_{i}(\omega) Z_{i}(\gamma) .
\end{gathered}
$$

Здесь случайные $\quad$ величины $\quad a_{i j}(\omega), \sigma_{i j}(\omega) \quad$ и $\quad a_{i}(\omega), \sigma_{i}(\omega) \quad$ независимы и имеют конечные математические ожидания, а нечеткие величины $Z_{i j}(\gamma)=\left[\underline{m}_{i j}, \bar{m}_{i j}, \underline{d}_{i j} \bar{d}_{i j}\right]_{L R}$ и $Z_{i}(\gamma)=\left[\underline{m}_{i}, \bar{m}_{i}, \underline{d}_{i} \bar{d}_{i}\right]_{L R}$ взаимно $T_{w}$-связаны.

\section{4. Эквивалентный стохастический аналог}

Согласно [4] задачу (1)-(2) можно свести к следующей задаче стохастического программирования:

$$
\begin{gathered}
\lambda_{0} m_{\mathrm{E}\left[f_{0}\right]}(x)+\beta_{0} d_{\mathrm{E}\left[f_{0}\right]}(x) \rightarrow \min _{x}, \\
\left\{\begin{array}{l}
\lambda_{i} m_{\mathrm{E}\left[f_{i}\right]}(x)+\beta_{i} d_{\mathrm{E}\left[f_{i}\right]}(x) \leq 0, i \in \overline{1, k} \\
x \in X .
\end{array}\right.
\end{gathered}
$$

Здесь в зависимости от $\mathcal{R}_{i}$ и $\tau_{i}$ для $i=\overline{0, k}$

$$
\begin{gathered}
\lambda_{i}= \pm 1, \beta_{i}=-L\left(\alpha_{i}\right),-L\left(1-\alpha_{i}\right), R\left(\alpha_{i}\right) \text { или } R\left(1-\alpha_{i}\right), \\
m_{\mathrm{E}\left[f_{i}\right]}(x)=\underline{m}_{\mathrm{E}\left[f_{i}\right]}(x) \text { или } \bar{m}_{\mathrm{E}\left[f_{i}\right]}(x), \quad d_{\mathrm{E}\left[f_{i}\right]}(x)=\underline{d}_{\mathrm{E}\left[f_{i}\right]}(x) \text { или } \bar{d}_{\mathrm{E}\left[f_{i}\right]}(x),
\end{gathered}
$$

при этом $m \leq k \leq 2 m$. Предположим, что

$$
\left.\bar{a}_{i j}=\mathrm{E}\left[a_{i j}(\omega)\right], \bar{\sigma}_{i j}=\mathrm{E}\left[\sigma_{i j}(\omega)\right], \quad \bar{a}_{i}=\mathrm{E}\left[a_{i}(\omega)\right], \quad \bar{\sigma}_{i}=\mathrm{E}\left[\sigma_{i} \omega\right)\right],
$$


тогда функции $\underline{m}_{\mathrm{E}\left[f_{i}\right]}(x), \bar{m}_{\mathrm{E}\left[f_{i}\right]}(x)$ и $\underline{d}_{\mathrm{E}\left[f_{i}\right]}(x), \bar{d}_{\mathrm{E}\left[f_{i}\right]}(x)$ примут вид

$$
\begin{gathered}
\underline{m}_{\mathrm{E}\left[f_{i}\right]}(x)=\sum_{j=1}^{n}\left(\bar{a}_{i j}+\bar{\sigma}_{i j} \underline{m}_{i j}\right) x_{j}-\left(\bar{a}_{i}+\bar{\sigma}_{i} \underline{m}_{i}\right), \\
\bar{m}_{\mathrm{E}\left[f_{i}\right]}(x)=\sum_{j=1}^{n}\left(\bar{a}_{i j}+\bar{\sigma}_{i j} \bar{m}_{i j}\right) x_{j}-\left(\bar{a}_{i}+\bar{\sigma}_{i} \bar{m}_{i}\right), \\
\underline{d}_{\mathrm{E}\left[f_{i}\right]}(x)=\mathrm{E}\left[\max \left\{\sigma_{i 1}(\omega) \underline{d}_{i 1} x_{1}, \cdots, \sigma_{i n}(\omega) \underline{d}_{i n} x_{n}, \sigma_{i}(\omega) \underline{d}_{i}\right\}\right], \\
\bar{d}_{\mathrm{E}\left[f_{i}\right]}(x)=\mathrm{E}\left[\max \left\{\sigma_{i 1}(\omega) \bar{d}_{i 1} x_{1}, \cdots, \sigma_{i n}(\omega) \bar{d}_{i n} x_{n}, \sigma_{i}(\omega) \bar{d}_{i}\right\}\right] .
\end{gathered}
$$

Стоит заметить, что функции $m_{\mathrm{E}\left[f_{i}\right]}(x)$ и $d_{\mathrm{E}\left[f_{i}\right]}(x)$ можно представить в следующем виде

$$
\begin{gathered}
m_{f_{i}}(x, \omega)=\sum_{j=1}^{n}\left(\bar{a}_{i j}(\omega)+\bar{\sigma}_{i j} m_{i j}\right) x_{j}-\left(\bar{a}_{i}(\omega)+\bar{\sigma}_{i} m_{i}\right), \\
d_{f_{i}}(x, \omega)=\max \left\{\sigma_{i 1}(\omega) d_{i 1} x_{1}, \cdots, \sigma_{i n}(\omega) d_{i n} x_{n}, \sigma_{i}(\omega) d_{i}\right\} .
\end{gathered}
$$

\section{5. Метод стохастических квазиградиентов}

В работах $[3,4]$ для решения задачи (3)-(4) был применен метод стохастических квазиградиентов, который является стохастическим вариантом градиентного метода Эрроу-Гурвица. Основная идея стохастических квазиградиентных методов заключается в том, что вместо градиентов или обобщенных градиентов при решении задачи используются их стохастические оценки, которые позволяют применять данные методы даже в случае, если функции ограничений и цели нельзя точно вычислить. Таким образом, применение данного подхода для решения задачи (3)-(4) позволит избежать трудоемких вычислений значений функций $d_{\mathrm{E}\left[f_{i}\right]}(x)$.

Кратко опишем основные шаги метода стохастических квазиградиентов [18]. Обозначим через $\hat{\varphi}_{x}(x, u)$ вектор обобщенного градиента функции Лагранжа $\varphi(x, u)$, отвечающей некоторой задаче выпуклого математического программирования, по переменным $x$ при фиксированном $u$, т. е. такой вектор, что для любого $z \in X$ выполняется

$$
\varphi(z, u)-\varphi(x, u) \geq\left\langle\widehat{\varphi}_{x}(x, u), z-x\right\rangle,
$$

а через $\varphi_{u}(x, u)$ - градиент этой функции по переменным $u$.

Далее строим последовательность точек $\left(x^{s}, u^{s}\right)$, исходя из следующих соотношений:

$$
\begin{aligned}
& x^{s+1}=\Pi_{X}\left(x^{s}-\rho_{s} \gamma_{s} \xi^{s}\right), s=0,1, \ldots, \\
& u^{s+1}=\Pi_{U}\left(u^{s}+\rho_{s} \gamma_{s} \zeta^{s}\right), s=0,1, \ldots,
\end{aligned}
$$

где $\left(x^{0}, u^{0}\right)$ - произвольное начальное приближение, $\rho_{s}$ - величина шага, $\gamma_{s}-$ нормирующий множитель; $\Pi_{X}$ - оператор проектирования на множество $X$, который ставит в соответствие каждой точке $x \in R^{n}$ точку $\Pi_{X}(x) \in X$ так, что

$$
\left\|y-\Pi_{X}(x)\right\| \leq\|y-x\|, \forall y \in X
$$


$\Pi_{U}$ - оператор проектирования на некоторое выпуклое и замкнутое множество $U$, содержащее компоненты $u^{*}$ седловых точек $\left(x^{*}, u^{*}\right)$ функции Лагранжа $\varphi(x, u)$ в области $x \in X, u \geqslant 0 ; \xi^{s}, \zeta^{s}-$ случайные векторы такие, что

$$
\begin{aligned}
\mathrm{E}\left[\xi^{s} \mid\left(x^{0}, u^{0}\right), \ldots,\left(x^{s}, u^{s}\right)\right] & =a_{s} \widehat{\varphi}_{x}\left(x^{s}, u^{s}\right)+b^{s}, \\
\mathrm{E}\left[\zeta^{s} \mid\left(x^{0}, u^{0}\right), \ldots,\left(x^{s}, u^{s}\right)\right] & =a_{s} \varphi_{u}\left(x^{s}, u^{s}\right)+d^{s},
\end{aligned}
$$

где случайная величина $a_{s} \geqslant 0$ и случайные векторы $b^{s}, d^{s}$ измеримы относительно $\sigma$-подалгебры $\mathcal{B}_{s}$, индуцированной семейством случайных величин $\left(x^{0}, u^{0}\right), \ldots$, $\left(x^{s}, u^{s}\right)$. Величины $\rho_{s}, \gamma_{s}$ также измеримы относительно $\mathcal{B}_{s}$.

Функция Лагранжа задачи (3)-(4) будет выглядеть следующим образом [4]:

$$
\varphi(x, u)=\lambda_{0} m_{\mathrm{E}\left[f_{0}\right]}(x)+\beta_{i} d_{\mathrm{E}\left[f_{0}\right]}(x)+\sum_{i=1}^{k} u_{i}\left(\lambda_{i} m_{\mathrm{E}\left[f_{i}\right]}(x)+\beta_{i} d_{\mathrm{E}\left[f_{i}\right]}(x)\right) .
$$

Определим вектор $\xi^{s}$ на s-й итерации следующим образом:

$$
\xi^{s}=\xi_{0}^{s}+\sum_{i=1}^{k} u_{i}^{s} \xi_{i}^{s}
$$

где

$$
\xi_{i}^{s}=\left(\lambda_{i}\left(\bar{a}_{i 1}+\bar{\sigma}_{i 1} m_{i 1}\right)+\beta_{i} t_{i 1}^{s} \sigma_{i 1}\left(\omega^{s}\right) d_{i 1}, \ldots, \lambda_{i}\left(\bar{a}_{i n}+\bar{\sigma}_{i n} m_{i n}\right)+\beta_{i} t_{i n}^{s} \sigma_{i n}\left(\omega^{s}\right) d_{i n}\right) .
$$

Здесь $x^{s}$ - точка, полученная на s-й итерации, $\sigma_{i j}\left(\omega^{s}\right)$ - реализация случайной величины $\sigma_{i j}(\omega)$ на s-й итерации; $t_{i j}^{s}=1$, если $j$ такой номер, что

$$
\sigma_{i j}\left(\omega^{s}\right) d_{i j} x_{i j}^{s} \geqslant \sigma_{i k}\left(\omega^{s}\right) d_{i k} x_{i k}^{s}
$$

для любых $k=\overline{1, n}$, в противном случае $t_{i j}^{s}=0$.

В качестве вектора $\zeta^{s}$ возьмем градиент функции Лагранжа $\varphi(x, u)$ по $u[4]$

$$
\zeta^{s}=\left(\lambda_{1} m_{\mathrm{E}\left[f_{1}\right]}(x)+\beta_{1} d_{\mathrm{E}\left[f_{1}\right]}(x), \ldots, \lambda_{k} m_{\mathrm{E}\left[f_{k}\right]}(x)+\beta_{k} d_{\mathrm{E}\left[f_{k}\right]}(x)\right) .
$$

В работе [4] показано, что если функция цели (3) и функции ограничений (4) являются непрерывными и выпуклыми, множество $X$ выпуклое и замкнутое, а ограничения (4) удовлетворяют условию Слейтера, то справедлива следующая теорема.

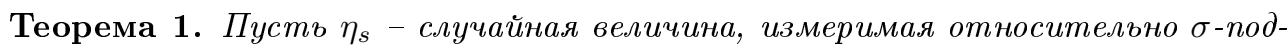
алгебры $\mathcal{B}_{s}$, индуцированной величинами $\left(x^{0}, u^{0}\right), \ldots,\left(x^{s}, u^{s}\right)$, такая, что

$$
\mathrm{E}\left[\left\|\xi^{s}\right\|^{2}+\left\|\zeta^{s}\right\|^{2} \mid\left(x^{0}, u^{0}\right), \ldots,\left(x^{s}, u^{s}\right)\right] \leqslant \eta_{s}^{2} \leqslant C_{L}<\infty
$$

при $\left\|x^{k}\right\|+\left\|u^{k}\right\| \leqslant L<\infty, k=\overline{0, s} ;$ нормирующий множитель $\gamma_{s}$ для некоторых чисел $\gamma, \bar{\gamma}$ удовлетворяет условию

$$
0<\underline{\gamma} \leqslant \gamma_{s} \eta_{s} \leqslant \bar{\gamma}<\infty
$$

величина $\rho_{s}$ такая, что

$$
\rho_{s} \geqslant 0, \quad \sum_{s=0}^{\infty} \rho_{s}=\infty, \quad \sum_{s=0}^{\infty} \rho_{s}^{2}<\infty
$$

Тогда с вероятностью 1 одна из предельных точек последовательности $x^{s}$ принадлежит $X^{*}$. 
Стоит отметить, что функции цели и функции ограничений задачи (3)-(4) являются выпуклыми, если $\beta_{i}=R\left(\alpha_{i}\right)$ или $R\left(1-\alpha_{i}\right)$, то есть если в исходной задаче возможностно-вероятностного программирования (1)-(2) в качестве $\tau_{i}$ была выбрана мера необходимости $\nu$.

\section{6. Упрощение теоремы о сходимости}

В этом пункте покажем, что, наложив дополнительное ограничение на случайные величины $\sigma_{i j}(\omega)$, можно опустить условия (5) и (6), тем самым упростив применение Теоремы 1 в реальных задачах.

Теорема 2. Пусть случайные величины $\sigma_{i j}(\omega)$ такие, что $\mathrm{E}\left[\sigma_{i j}^{2}(\omega)\right]<\infty$, тогда для всех $s=0,1, \ldots$ выполняется

$$
\mathrm{E}\left[\left\|\xi^{s}\right\|^{2}+\left\|\zeta^{s}\right\|^{2} \mid\left(x^{0}, u^{0}\right), \ldots,\left(x^{s}, u^{s}\right)\right]<\infty .
$$

Доказательство. Для упрощения дальнейших выкладок обозначим $\sigma$-алгебру, индуцированную величинами $\left(x^{0}, u^{0}\right), \ldots,\left(x^{s}, u^{s}\right)$, как $\mathcal{B}_{s}$, а вектор $\xi^{s}$ представим в следующем виде

$$
\xi^{s}=\sum_{i=0}^{k} u_{i}^{s} \xi_{i}^{s}
$$

где $u_{0}^{s}=1$ для всех $s=0,1, \ldots$

По свойствам математического ожидания имеем

$$
\mathrm{E}\left[\left\|\xi^{s}\right\|^{2}+\left\|\zeta^{s}\right\|^{2} \mid \mathcal{B}_{s}\right]=\mathrm{E}\left[\left\|\xi^{s}\right\|^{2} \mid \mathcal{B}_{s}\right]+\mathrm{E}\left[\left\|\zeta^{s}\right\|^{2} \mid \mathcal{B}_{s}\right] .
$$

Учитывая вид вектора $\xi^{s}$, можно получить, что

$$
\begin{aligned}
\left\|\xi^{s}\right\|^{2}=\left\|\sum_{i=0}^{k} u_{i}^{s} \xi_{i}^{s}\right\|^{2}=\sum_{j=1}^{n}\left(\sum_{i=0}^{k} u_{i}^{s} \xi_{i j}^{s}\right)^{2} & =\sum_{j=1}^{n}\left(\sum_{i=0}^{k}\left(u_{i}^{s}\right)^{2}\left(\xi_{i j}^{s}\right)^{2}+2 \sum_{\substack{i, l=0, i \neq l}}^{k} u_{i}^{s} u_{l}^{s} \xi_{i j}^{s} \xi_{l j}^{s}\right) \\
& =\sum_{i=0}^{k}\left(u_{i}^{s}\right)^{2} \sum_{j=1}^{n}\left(\xi_{i j}^{s}\right)^{2}+\sum_{\substack{i, l=0, i \neq l}}^{k} u_{i}^{s} u_{l}^{s} \sum_{j=1}^{n} \xi_{i j}^{s} \xi_{l j}^{s} .
\end{aligned}
$$

Здесь $\xi_{i j}^{s}-j$ компонента вектора $\xi_{i}^{s}$. Тогда

$$
\mathrm{E}\left[\left\|\xi^{s}\right\|^{2} \mid \mathcal{B}_{s}\right]=\sum_{i=0}^{k}\left(u_{i}^{s}\right)^{2} \sum_{j=1}^{n} \mathrm{E}\left[\left(\xi_{i j}^{s}\right)^{2} \mid \mathcal{B}_{s}\right]+\sum_{\substack{i, l=0, i \neq l}}^{k} u_{i}^{s} u_{l}^{s} \sum_{j=1}^{n} \mathrm{E}\left[\xi_{i j}^{s} \xi_{l j}^{s} \mid \mathcal{B}_{s}\right] .
$$

Лемма 1. Пусть случайные величины $a_{i j}(\omega), \sigma_{i j}(\omega)$ u $\sigma_{i j}^{2}(\omega)$ имеют конечные математические ожидания, тогда справедливо неравенство $\mathrm{E}\left[\left(\xi_{i j}^{s}\right)^{2} \mid \mathcal{B}_{s}\right]<\infty$.

Доказательство. Действительно

$$
\begin{aligned}
\left(\xi_{i j}^{s}\right)^{2} & =\left(\lambda_{i}\left(\bar{a}_{i j}+\bar{\sigma}_{i j} m_{i j}\right)+\beta_{i} t_{i j}^{s} \sigma_{i j}\left(\omega^{s}\right) d_{i j}\right)^{2} \\
& =\left(\bar{a}_{i j}+\bar{\sigma}_{i j} m_{i j}\right)^{2}+\beta_{i}^{2} t_{i j}^{s} \sigma_{i j}^{2}\left(\omega^{s}\right) d_{i j}^{2}+2 \lambda_{i}\left(\bar{a}_{i j}+\bar{\sigma}_{i j} m_{i j}\right) \beta_{i} t_{i j}^{s} \sigma_{i j}\left(\omega^{s}\right) d_{i j} .
\end{aligned}
$$


Таким образом

$$
\begin{aligned}
\mathrm{E}\left[\left(\xi_{i j}^{s}\right)^{2} \mid \mathcal{B}_{s}\right]=\left(\bar{a}_{i j}+\bar{\sigma}_{i j} m_{i j}\right)^{2}+\beta_{i}^{2} t_{i j}^{s} \mathrm{E}[ & \left.\sigma_{i j}^{2}\left(\omega^{s}\right) \mid \mathcal{B}_{s}\right] d_{i j}^{2} \\
& +2 \lambda_{i}\left(\bar{a}_{i j}+\bar{\sigma}_{i j} m_{i j}\right) \beta_{i} t_{i j}^{s} \mathrm{E}\left[\sigma_{i j}\left(\omega^{s}\right) \mid \mathcal{B}_{s}\right] d_{i j}
\end{aligned}
$$

Так как математические ожидания $a_{i j}(\omega), \sigma_{i j}(\omega)$ и $\sigma_{i j}^{2}(\omega)$ конечны, то легко видеть, что и $\mathrm{E}\left[\left(\xi_{i j}^{s}\right)^{2} \mid \mathcal{B}_{s}\right]$ также конечно.

Лемма 2. Пусть случайные величины $a_{i j}(\omega)$ u $\sigma_{i j}(\omega)$ имеют конечные математические ожидания, тогда справедливо неравенство $\mathrm{E}\left[\xi_{i j}^{s} \xi_{l j}^{s} \mid \mathcal{B}_{s}\right]<\infty$.

Доказательство. Действительно

$$
\begin{aligned}
\xi_{i j}^{s} \xi_{l j}^{s} & =\left(\lambda_{i}\left(\bar{a}_{i j}+\bar{\sigma}_{i j} m_{i j}\right)+\beta_{i} t_{i j}^{s} \sigma_{i j}\left(\omega^{s}\right) d_{i j}\right)\left(\lambda_{l}\left(\bar{a}_{l j}+\bar{\sigma}_{l j} m_{l j}\right)+\beta_{l} t_{l j}^{s} \sigma_{l j}\left(\omega^{s}\right) d_{l j}\right) \\
& =\lambda_{i} \lambda_{l}\left(\bar{a}_{i j}+\bar{\sigma}_{i j} m_{i j}\right)\left(\bar{a}_{l j}+\bar{\sigma}_{l j} m_{l j}\right)+\beta_{i} \beta_{l} t_{i j}^{s} t_{l j}^{s} \sigma_{i j}\left(\omega^{s}\right) \sigma_{l j}\left(\omega^{s}\right) d_{i j} d_{l j} \\
& +\lambda_{i}\left(\bar{a}_{i j}+\bar{\sigma}_{i j} m_{i j}\right) \beta_{l} t_{l j}^{s} \sigma_{l j}\left(\omega^{s}\right) d_{l j}+\lambda_{l}\left(\bar{a}_{l j}+\bar{\sigma}_{l j} m_{l j}\right) \beta_{i} t_{i j}^{s} \sigma_{i j}\left(\omega^{s}\right) d_{i j} .
\end{aligned}
$$

Отсюда следует, что

$$
\begin{aligned}
\mathrm{E}\left[\xi_{i j}^{s} \xi_{l j}^{s} \mid \mathcal{B}_{s}\right]= & \lambda_{i} \lambda_{l}\left(\bar{a}_{i j}+\bar{\sigma}_{i j} m_{i j}\right)\left(\bar{a}_{l j}+\bar{\sigma}_{l j} m_{l j}\right) \\
& +\beta_{i} \beta_{l} t_{i j}^{s} t_{l j}^{s} \mathrm{E}\left[\sigma_{i j}\left(\omega^{s}\right) \mid \mathcal{B}_{s}\right] \mathrm{E}\left[\sigma_{l j}\left(\omega^{s}\right) \mid \mathcal{B}_{s}\right] d_{i j} d_{l j} \\
& +\lambda_{i}\left(\bar{a}_{i j}+\bar{\sigma}_{i j} m_{i j}\right) \beta_{l} t_{l j}^{s} \mathrm{E}\left[\sigma_{i j}\left(\omega^{s}\right) \mid \mathcal{B}_{s}\right] d_{l j} \\
& +\lambda_{l}\left(\bar{a}_{l j}+\bar{\sigma}_{l j} m_{l j}\right) \beta_{i} t_{i j}^{s} \mathrm{E}\left[\sigma_{l j}\left(\omega^{s}\right) \mid \mathcal{B}_{s}\right] d_{i j} .
\end{aligned}
$$

Легко видеть, что конечность математических ожиданий величин $a_{i j}(\omega)$ и $\sigma_{i j}(\omega)$ ведет к конечности $\mathrm{E}\left[\xi_{i j}^{s} \xi_{l j}^{s} \mid \mathcal{B}_{s}\right]$.

С учетом доказанных лемм, несложно показать, что $\mathrm{E}\left[\left\|\xi^{s}\right\|^{2} \mid \mathcal{B}_{s}\right]<\infty$. С другой стороны

$$
\begin{aligned}
\mathrm{E}\left[\left\|\zeta^{s}\right\|^{2} \mid \mathcal{B}_{s}\right] & =\mathrm{E}\left[\sum_{i=1}^{k}\left(\lambda_{i} m_{\mathrm{E}\left[f_{i}\right]}\left(x^{s}\right)+\beta_{i} d_{\mathrm{E}\left[f_{i}\right]}\left(x^{s}\right)\right)^{2} \mid \mathcal{B}_{s}\right] \\
& =\sum_{i=1}^{k}\left(\lambda_{i} m_{\mathrm{E}\left[f_{i}\right]}\left(x^{s}\right)+\beta_{i} d_{\mathrm{E}\left[f_{i}\right]}\left(x^{s}\right)\right)^{2}
\end{aligned}
$$

Так как функции $\lambda_{i} m_{\mathrm{E}\left[f_{i}\right]}(x)+\beta_{i} d_{\mathrm{E}\left[f_{i}\right]}(x)$ непрерывны, а множество $X$ ограничено, то очевидно, что и $\mathrm{E}\left[\left\|\zeta^{s}\right\|^{2} \mid \mathcal{B}_{s}\right]$ также будет ограничено.

Таким образом при условиях, когда функция цели (3) и функции ограничений (4) являются непрерывными и выпуклыми, множество $X$ выпуклым и замкнутым, а ограничения (4) удовлетворяют условию Слейтера, будет справедлива следующая теорема.

Теорема 3. Пусть случайные величины $\sigma_{i j}(\omega)$ такие, что $\mathrm{E}\left[\sigma_{i j}^{2}(\omega)\right]<\infty$; нормирующий множитель $\gamma_{s}$ для некоторых чисел $\underline{\gamma}, \bar{\gamma}$ удовлетворяет условию

$$
0<\underline{\gamma} \leqslant \gamma_{s} \leqslant \bar{\gamma}<\infty
$$


величина $\rho_{s}$ такая, что

$$
\rho_{s} \geqslant 0, \quad \sum_{s=0}^{\infty} \rho_{s}=\infty, \quad \sum_{s=0}^{\infty} \rho_{s}^{2}<\infty .
$$

Тогда с вероятностью 1 одна из предельных точек последователъности $x^{s}$ принадлежит $X^{*}$.

Стоит отметить, что требование конечности математических ожиданий случайных величин $\sigma_{i j}^{2}(\omega)$ достаточно легко обеспечить в реальных задачах.

\section{Заключение}

В статье рассмотрена модель возможностно-вероятностного программирования с ограничениями по возможности и необходимости в случае слабейшей tнормы, построен ее стохастические аналог и обоснован метод решения, основанный на стохастических квазиградиентах.

Также было показано, что теорема о сходимости метода решения поставленной задачи возможностно-вероятностного программирования, основанного на методе стохастических квазиградиентов [4], остается верной, если условие об ограниченности математического ожидания суммы квадратов норм квазиградиентов (5) заменить на ограниченность математических ожиданий случайных величин $\sigma_{i j}^{2}(\omega)$, что проще обеспечить в реальных задачах, например, таких как задача оптимизации инвестиционного портфеля в условиях гибридной неопределенности.

В плане дальнейших исследований представляется необходимым получение оценок вычислительной сложности примененного метода стохастических квазиградиентов, а также сравнение его с непрямыми методами решения задач возможностно-вероятностной оптимизации.

\section{Список литературы}

[1] Yazenin A.V. Linear programming with random fuzzy data // Soviet Journal of Computer and Systems Sciences. 1991. № 30. Pp. 86-93.

[2] On a method of solving a problem of linear programming with random fuzzy data // Journal of Computer and Systems Sciences International. 1997. Vol. 36, № 5. Pp. 737-741.

[3] Егорова Ю.Е., Язенин А.В. Стохастический квазиградиентный метод решения задач возможностно-вероятностной оптимизации одного класса // Вестник ТвГУ. Серия: Прикладная математика. 2014. № 4. С. 57-70.

[4] Егорова Ю.Е., Язенин А.В. К проблеме возможностно-вероятностной оптимизации // Известия Российской Академии наук. Теория и системы управления. 2017. № 4. С. 104-120.

[5] Yazenin A., Soldatenko I. On the Problem of Possibilistic-Probabilistic Optimization with Constraints on Possibility/Probability // Advances in Intelligent Systems and Computing. Eds. by Giove S., Masulli F., Fuller R.. Series: Lecture Notes in Computer Science. Vol. 11291. Cham: Springer, 2019. Pp. 43-54. 
[6] Yazenin A., Soldatenko I. On the Problem of Possibilistic-Probabilistic Optimization with Constraints on Possibility/Probability // Advances in Intelligent Systems and Computing. Eds. by Giove S., Masulli F., Fuller R.. Series: Lecture Notes in Computer Science. Vol. 11291. Cham: Springer, 2019. Pp. 43-54.

[7] Егорова Ю.Е. Стохастический метод штрафов в задачах возможностновероятностного программирования // Нечеткие системы и мягкие вычисления. 2019. Т. 14, № 1. С. 64-74. https://doi.org/10.26456/fssc53

[8] Yazenin A., Soldatenko I. A portfolio of minimum risk in a hybrid uncertainty of a possibilistic-probabilistic type: comparative study // Advances in Fuzzy Logic and Technology 2017 (EUSFLAT 2017, IWIFSGN 2017). Eds. by Kacprzyk J., Szmidt E., Zadrozny S., Atanassov K., Krawczak M.. Series: Advances in Intelligent Systems and Computing. Vol. 643. 2018. Pp. 551-563. https://doi.org/10.1007/978-3-319-66827-7_51

[9] Egorova Yu.E., Yazenin A.V. A method for minimum risk portfolio optimization under hybrid uncertainty // Journal of Physics: Conference Series. Vol. 973. 2018. ID 012033.

[10] Nahmias S. Fuzzy variables // Fuzzy Sets and Systems. 1978. Vol. 1, № 2. Pp. 97110. https://doi.org/10.1016/0165-0114(78)90011-8

[11] Nahmias S. Fuzzy variables in a random environment // Advances in Fuzzy Set Theory and Applications. Eds. by M.M. Gupta, R.K. Ragade, R.R. Yager. Amsterdam: North-Holland, 1979. Pp. 165-180.

[12] Yazenin A., Wagenknecht M. Possibilistic Optimization. A Measure-Based Approach. Cotbus, Germany: Brandenburgische Technische Universitat, 1996. $133 \mathrm{p}$.

[13] Язенин А.В. Основные понятия теории возможностей. Математический аппарат для принятия решений в условиях гибридной неопределенности. М.: Физматлит, 2016. 144 с.

[14] Хохлов М.Ю., Язенин А.В. Расчет числовых характеристик нечетких случайных величин // Вестник ТвГУ. Серия: Прикладная математика. 2003. № 1 . С. 39-43.

[15] Дюбуа Д., Прад А. Теория возможностей. М.: Радио и связь, 1990.

[16] Nguyen H.T., Walker E.A. A First Course in Fuzzy Logic. Boca Raton: CRC Press, 1997.

[17] Hong D.H. Parameter estimations of mutually t-related fuzzy variables // Fuzzy Sets and Systems. 2001. Vol. 123, № 1. Pp. 63-71. https://doi.org/10.1016/S01650114(00)00113-5

[18] Ермольев Ю.М. Методы стохастического программирования. М.: Наука, 1976. 


\section{Образец цитирования}

Егорова Ю.Е. К методу решения задач возможностно-вероятностного программирования // Нечеткие системы и мягкие вычисления. 2021. Т. 16, № 1 . C. 21-33. https://doi.org/10.26456/fssc77

\section{Сведения об авторах}

1. Егорова Юлия Евгеньевна

ассистент кафедры информационных технологий Тверского государственного университета.

Россия, 170100, г. Тверь, ул. Желябова, д. З3, ТвГУ.

E-mail: j.e.egorova@gmail.com 


\title{
ON A METHOD OF SOLVING OF POSSIBILISTIC-PROBABILISTIC PROGRAMMING PROBLEMS
}

\author{
Egorova Yulia Evgenyevna \\ Assistant in the Department of Information Technologies, Tver State University \\ Russia, 170100, Tver, Zhelyabova str., 33, TverSU. \\ E-mail: j.e.egorova@gmail.com
}

\begin{abstract}
Received 25.12.2020, revised 06.05.2021.
The paper studies possibilistic-probabilistic optimization problems, based on the principle of expected possibility, and a method for solving its stochastic analogue in the case of the weakest t-norm describing the interaction of fuzzy parameters. The conditions that are easier to verify and ensure the convergence of the method of stochastic quasigradients of the solution of an equivalent stochastic analog are obtained.
\end{abstract}

Keywords: possitbilistic-probabilistic optimization, stochastic quasigradient method, fuzzy random variable, the weakest t-norm.

\section{Citation}

Egorova Yu.E., "On a method of solving of possibilistic-probabilistic programming problems", Nechetkie Sistemy i Myagkie Vychisleniya [Fuzzy Systems and Soft Computing], 16:1 (2021), 21-33(in Russian). https://doi.org/10.26456/fssc77

\section{References}

[1] Yazenin A.V., "Linear programming with random fuzzy data", Soviet Journal of Computer and Systems Sciences, 1991, № 30, 86-93.

[2] "On a method of solving a problem of linear programming with random fuzzy data", Journal of Computer and Systems Sciences International, 36:5 (1997), $737-741$.

[3] Egorova Yu.E., Yazenin A.V., "Stochastic quasi-gradient method for solving possibilistic-probabilistic optimization task of one class", Vestnik TvGU. Seriya: Prikladnaya Matematika [Herald of Tver State University. Series: Applied Mathematics], 2014, № 4, 57-70 (in Russian).

[4] Egorova Y.E., Yazenin A.V., "The problem of possibilistic-probabilistic optimization", Journal of Computer and Systems Sciences International, 56:4 (2017), 652-667.

[5] Yazenin A., Soldatenko I., "On the Problem of Possibilistic-Probabilistic Optimization with Constraints on Possibility/Probability", Advances in Intelligent Systems and Computing. V.11291, Lecture Notes in Computer Science, eds. Giove S., Masulli F., Fuller R., Springer, Cham, 2019, 43-54. 
[6] Yazenin A., Soldatenko I., "On the Problem of Possibilistic-Probabilistic Optimization with Constraints on Possibility/Probability", Advances in Intelligent Systems and Computing. V.11291, Lecture Notes in Computer Science, eds. Giove S., Masulli F., Fuller R., Springer, Cham, 2019, 43-54.

[7] Egorova Yu.E., "Stochastic penalty method in problems of probabilisticprobabilistic programming", Nechetkie Sistemy i Myagkie Vychisleniya [Fuzzy Systems and Soft Computing], 14:1 (2019), 64-74 (in Russian), https://doi.org/10.26456/fssc53.

[8] Yazenin A., Soldatenko I., "A portfolio of minimum risk in a hybrid uncertainty of a possibilistic-probabilistic type: comparative study", Advances in Fuzzy Logic and Technology 201\%. V. 643 (EUSFLAT 2017, IWIFSGN 2017), Advances in Intelligent Systems and Computing, eds. Kacprzyk J., Szmidt E., Zadrozny S., Atanassov K., Krawczak M., 2018, 551-563, https://doi.org/10.1007/978-3-31966827-7_51.

[9] Egorova Yu.E., Yazenin A.V., "A method for minimum risk portfolio optimization under hybrid uncertainty", Journal of Physics: Conference Series. V.973, 2018.

[10] Nahmias S., "Fuzzy variables", Fuzzy Sets and Systems, 1:2 (1978), 97-110, https://doi.org/10.1016/0165-0114(78)90011-8.

[11] Nahmias S., "Fuzzy variables in a random environment", Advances in Fuzzy Set Theory and Applications, eds. M.M. Gupta, R.K. Ragade, R.R. Yager, NorthHolland, Amsterdam, 1979, 165-180.

[12] Yazenin A., Wagenknecht M., Possibilistic Optimization. A Measure-Based Approach, Brandenburgische Technische Universitat, Cotbus, Germany, 1996, 133 pp.

[13] Yazenin A.V., Osnovnye ponyatiya teorii vozmozhnostej. Matematicheskij apparat dlya prinyatiya reshenij $v$ usloviyakh gibridnoj neopredelennosti [Basic concepts of the theory of possibilities. Mathematical decision-making apparatus in a hybrid uncertainty], Fizmatlit Publ., Moscow, 2016 (in Russian), 144 pp.

[14] Khokhlov M.Yu., Yazenin A.V., "Calculation of numerical characteristics of fuzzy random variables", Vestnik TvGU. Seriya: Prikladnaya Matematika [Herald of Tver State University. Series: Applied Mathematics], 2003, № 1, 39-43 (in Russian).

[15] Dubois D., Prade H., Theorie des Possibilites, Application a la Representation des Connaissances en Informatique, Masson, Paris, 1988.

[16] Nguyen H.T., Walker E.A., A First Course in Fuzzy Logic, CRC Press, Boca Raton, 1997.

[17] Hong D.H., "Parameter estimations of mutually t-related fuzzy variables", Fuzzy Sets and Systems, 123:1 (2001), 63-71, https://doi.org/10.1016/S01650114(00)00113-5.

[18] Ermolev Yu.M., Metody stokhasticheskogo programmirovaniya [Stochastic programming methods], Nauka Publ., Moscow, 1976 (in Russian). 